\title{
Communication
}

\section{A Standard Model Neutrino Oscillation}

\author{
Robert B. Hayes ${ }^{1}$
}

1 North Carolina State University 1; rbhayes@ncsu.edu

\begin{abstract}
This work argues the mass term in the neutrino wavefunction propagator is due to entanglement with its associated origins. The implication being that neutrino flavor is conserved in weak processes and shared among all particles emanating from the last interaction with a nucleon. In so doing, the neutrino mass propagator is real but not ascribed to the neutrino outside of entanglement. The proposed theory will be readily testable in that reactor and solar neutrinos will oscillate but both muon decay neutrinos and accelerator neutrinos created by pure lepton interactions will not oscillate..
\end{abstract}

Keywords: neutrino; standard model; entanglement; flavor conservation

\section{Introduction}

The existence of experimentally measured neutrino oscillations [1] has resulted in a consensus that neutrinos cannot have a zero rest mass [2,3] giving rise to the expectation that new physics outside the standard model must be real. Other models exist such as faster than light [4], sterile neutrinos [5] along with other explanatory theories postulating physics outside the standard model [6,7]. The initial postulate of the neutrino existence was itself just a means to attempt justification of retaining energy conservation in beta decay (ref) similar to the initial proposition of color charge being a means to justify consistency of the Pauli exclusion principle for otherwise identical quark states (ref). With similar inspiration, a model is proposed to maintain the standard model of the neutrino by assuming the neutrino is entangled with a nucleon where the nucleon retains a neutrino flavor which itself evolves in time giving rise to observed neutrino oscillations through entanglement.

The basic model for neutrino oscillations can be reduced to the form [8] $\boldsymbol{P}_{\boldsymbol{v}_{\boldsymbol{\alpha}} \rightarrow \boldsymbol{v}_{\boldsymbol{\beta}}}=$ $\sum_{k l}\left\langle\boldsymbol{v}_{\boldsymbol{\beta}} \mid \boldsymbol{v}_{\boldsymbol{k}}\right\rangle\left\langle\boldsymbol{v}_{\boldsymbol{k}} \mid \boldsymbol{v}_{\boldsymbol{\alpha}}\right\rangle\left\langle\boldsymbol{v}_{\boldsymbol{l}} \mid \boldsymbol{v}_{\boldsymbol{\beta}}\right\rangle\left\langle\boldsymbol{v}_{\boldsymbol{\alpha}} \mid \boldsymbol{v}_{l}\right\rangle \boldsymbol{e}^{-i \frac{\left(m_{k}-m_{l}\right)}{\gamma} t}$. Here, $\boldsymbol{\alpha}, \boldsymbol{\beta} \in\{\boldsymbol{e}, \boldsymbol{\mu}, \boldsymbol{\tau}\}, \boldsymbol{\alpha} \neq \boldsymbol{\beta}$, where the wavefunction is given by $\left|\boldsymbol{\Psi}_{\boldsymbol{v}}(\boldsymbol{t})\right\rangle=\boldsymbol{C}_{\boldsymbol{e}}(\boldsymbol{t})\left|\boldsymbol{v}_{\boldsymbol{e}}\right\rangle+\boldsymbol{C}_{\boldsymbol{\mu}}(\boldsymbol{t})\left|\boldsymbol{v}_{\boldsymbol{\mu}}\right\rangle+\boldsymbol{C}_{\tau}(\boldsymbol{t})\left|\boldsymbol{v}_{\tau}\right\rangle$ with the individual component amplitudes given by $\boldsymbol{C}_{\xi}(\boldsymbol{t})=$ $\sum_{j}\left[\sum_{k} \boldsymbol{e}^{-\boldsymbol{i m} \boldsymbol{m}_{\boldsymbol{k}} \boldsymbol{t}} \boldsymbol{U}_{\xi, \boldsymbol{k}} \boldsymbol{U}_{\boldsymbol{j} \boldsymbol{k}}^{*}\right] \boldsymbol{C}_{\boldsymbol{j}}(\mathbf{0})$. The unitary matrices for 2 flavors is simply $\boldsymbol{U}=$ $\left(\begin{array}{cc}\cos \theta & \sin \theta \\ -\sin \theta & \cos \theta\end{array}\right)$. In the 3 flavor consideration as done here, the mixing matrix can then be approximated (at small mixing angles $\boldsymbol{\theta}_{\mathbf{J}} \mathbf{k}$ ) from the PMNS model as $\boldsymbol{U}_{\boldsymbol{v}}=$ $\left(\begin{array}{ccc}1 & s_{12} & s_{13} e^{-i \delta} \\ -s_{12} & 1 & s_{23} \\ -s_{13} e^{-i \delta} & -s_{23} & 1\end{array}\right)+O\left(s_{j k}^{2}\right)$ where $s_{j k}=\sin \theta_{j k}$ and and $U_{j k} \rightarrow$ $\left[\boldsymbol{U}_{\boldsymbol{j} \boldsymbol{k}} \mid \boldsymbol{e}^{-\boldsymbol{i} \boldsymbol{\delta}_{\boldsymbol{j} \boldsymbol{k}}}\right.$ for the imaginary portions. The mass terms $m_{k}$ appearing in the $\boldsymbol{C}_{\boldsymbol{\alpha}}(\boldsymbol{t})$ amplitudes are invariably ascribed to the neutrino rest mass energy rather than being viewed as a phase parameter. This work explores the possibility that the mass terms relate to the properties of the combined system of the neutrino with its origin in such a way that the neutrino remains massless with the flavor variations being shared among the associated emergent fermions which force these changes in the measured neutrino via entanglement. 


\section{Theory}

A familiar means to create neutrinos through the standard Fermi diagrams are shown in Figure 1 (with pion decay in Fig 1A and muon decay in Fig 1B), we are comfortable that this explains experimental results. Here the pion or muon would have originated in a proton collision interaction for accelerated based oscillation experiments. The historical view on neutrino chirality from the standard model had initially believed that all $v_{\alpha}$ were left handed and $\bar{v}_{\alpha}$ were right handed [9]. With the recognition that flavor dynamics require particle mass terms in the wavefunction definition, the existence of flavor superposition's in the mass eigenstates has historically simply been assumed rather than allowed to arise from an entanglement response.
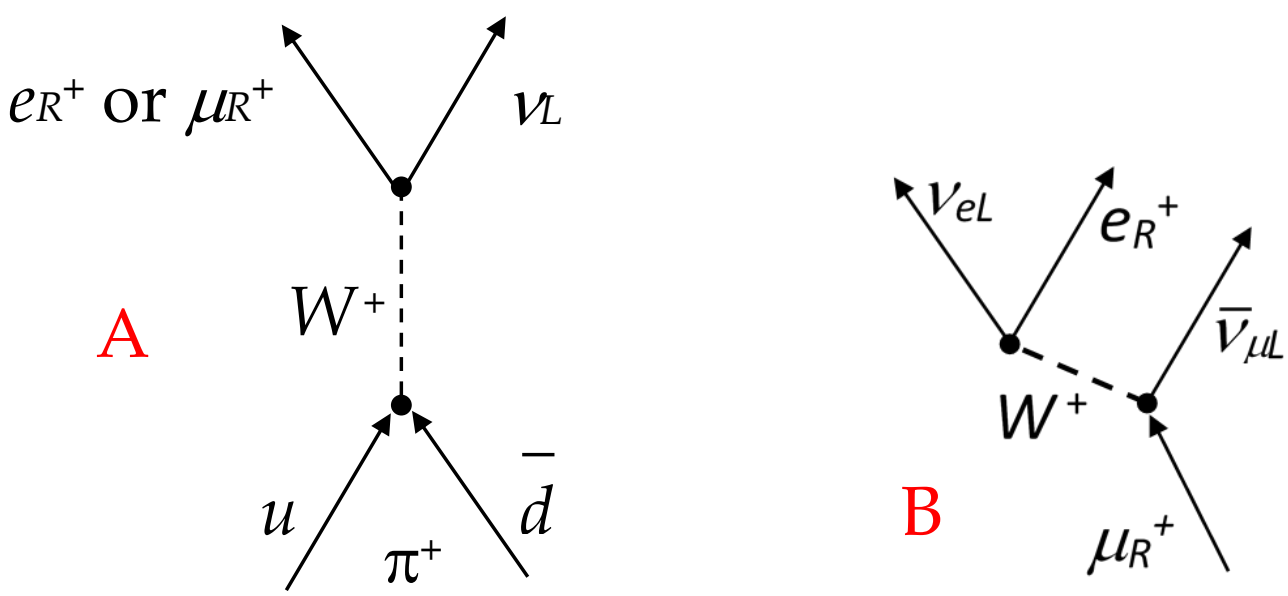

Figure 1. Feynman diagram for a characteristic pion decay followed by a potential muon creation and its decay. Fig $1 \mathrm{~A}$ on the left shows the initial pion decay with the optional muon from the pion decay reaction shown itself decaying in Fig. $1 \mathrm{~B}$ on the right.

The key tenet in the proposed theory here is that in measuring the flavor of any one neutrino, this drives compensatory flavor changes in the associated particles coming out of the various generation processes from which it originated, preserving a neutrino flavor conservation. The neutrino flavor is then carried by the pion or muon in Figure 1 respectively allowing transfer to the neutrino which is entangled with the initial proton (or neutron) interaction creating the pion or muon.

Here, the initial proton or neutron wavefunction which gave rise to the eventual neutrino birth is then represented by $\left|\Psi_{p, n}(t)\right\rangle=\left(C_{e}(t)+C_{\mu}(t)+C_{\tau}(t)\right)|p, n\rangle$. The entanglement requirement can then be imposed that $\left\langle\Psi_{v}(t) \mid \Psi_{n, p}(t)\right\rangle=$ $\langle v \mid n, p\rangle$ where $n, p$ is simply the neutron or proton originator and so the oscillations are no longer a time dependent value when both are measured.

As another example, standard positron decay in nuclear fusion as shown in Figure 2A would have its $v_{e L}$ oscillate with the down quark in the neutron from which the $\mathrm{W}^{+}$boson emanated. This particular scenario would require the probability of flavor transition for the down quark in the neutron to follow $P_{n_{\beta} \rightarrow n_{e}}=P_{v_{e} \rightarrow v_{\beta}}$ which then defines the initial flavor of the interacting down quark where $\beta \in\{\mu, \tau\}$. If such a neutron were found to decay after creation in an accelerator, the emergent proton and antineutrino would again share the flavor changes so that all particles 
would have to be measured to test $P_{v_{e} \rightarrow v_{\beta}}=P_{p_{\beta} \rightarrow p_{e}}$ for the reaction in Fig $2 \mathrm{~A}$ and $P_{\bar{v}_{e} \rightarrow \bar{v}_{\beta}}=P_{p_{\beta} \rightarrow p_{e}}$ for the reaction in Fig 2B.
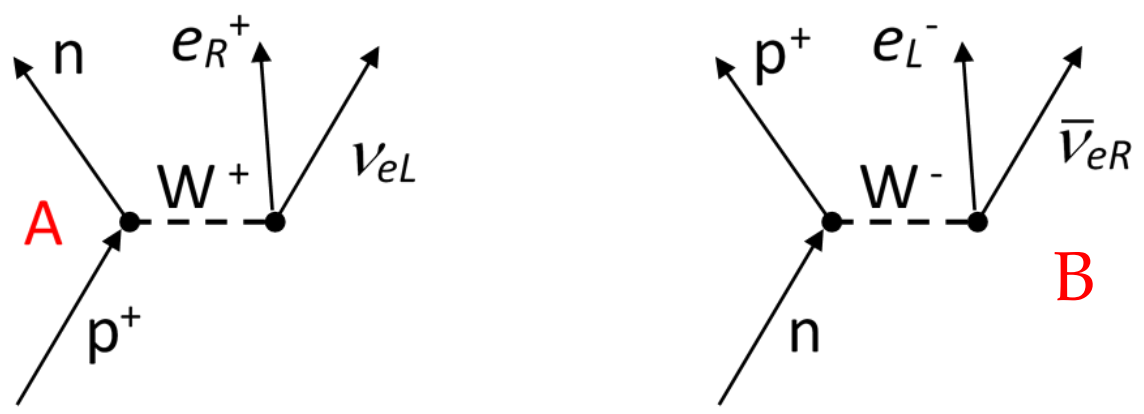

Figure 2. Fig 2A (left) shows the conversion of an up to a down quark (requisite for hydrogen fusion into deuterium). Fig 2B shows basic neutron beta decay.

Alternatively, if the antineutrino from Fig 2B were measured, the probability for the proton flavor could be probed to again test only $P_{\bar{v}_{e} \rightarrow \bar{v}_{\beta}}=P_{p_{\beta} \rightarrow p_{e}}$. In short, an essential test of neutrino flavor conservation will be a flavor measurement of all emanating particles at simultaneous delay periods.

If the probability of flavor transition of the emergent left handed muon antineutrino in Fig. 1B is given by the simplifying approximation [8] $P_{v_{\mu} \rightarrow v_{e}}=\sin ^{2} 2 \theta \sin ^{2} \frac{\Delta m^{2} L}{4 E_{0}}$, then the probability of flavor transition in the left handed electron neutrino coming from the same decay process would then be approximated by $P_{v_{e} \rightarrow v_{\beta}}=1-P_{v_{\mu} \rightarrow v_{e}}-P_{v_{\mu} \rightarrow v_{\tau}}$.

The mass term $\Delta m$ used in this probability has been associated with the quantum mechanical oscillation property of standard particles being left handed excluding the Dirac mass Lagrangian [10] $\mathcal{L}^{D}=\sum_{\alpha, \beta=e, \mu, \tau} \bar{v}_{\alpha L} M_{\alpha \beta}^{D} v_{\beta R}$. Rather, even when accepting only the Majorana version ${ }^{11}$ $\mathcal{L}^{M}=-0.5 \sum_{\alpha, \beta=e, \mu, \tau} \bar{v}_{\alpha L} M_{\alpha \beta}^{M} v_{\beta L}$ the requisite mass term remains. Here, the mass terms for the various flavor oscillations $M_{\alpha \beta}$, are assumed nonzero from which an isolated neutrino then has a time dependence of $\left|v_{\alpha}(t)\right\rangle=e^{-i m_{\alpha} t}\left|v_{\alpha}(0)\right\rangle$ using the standard convention of $c=h=1$. That the propagator in these units has dimensionality of mass does not require that the neutrinos have mass a priori when the matrix is $M_{\alpha \beta}$ effectively just a fit to the measurement data. The implication being that the mass corresponds to the nucleon and forces a neutron oscillation through entanglement only allowing all neutrinos to remain left handed and so consistent with the standard model [9].

Another way to consider this mechanism would be to cast the mass terms in units of $\mathrm{eV} \mathrm{c}^{-2}$, then for neutrino phase equivalent estimates of [12] 0.071, 0.072 and $0.087 \mathrm{eV}$, this would allow us to write $C_{\xi}(t)=\sum_{j}\left(e^{-i 0.071 t} U_{\xi, 1} U_{j, 1}^{*}+e^{-i 0.072 t} U_{\xi, 2} U_{j, 2}^{*}+e^{-i 0.087 t} U_{\xi, 3} U_{j, 3}^{*}\right) C_{j}(0)$. Again, this in no way is evidence for or against a neutrino mass.

\section{Test Options}

With decay scheme in Fig. 1, all emanating neutrinos will conserve flavor, collectively with their initiating nucleon. This offers a test mechanism but does require correlating each emergent neutrino along with its creation ejecta. Reactor and solar neutrinos are not operationally practical to track their progenitor dynamics but accelerator neutrinos may allow testing this theory.

The proton beams from the T2K [13], NOvA [14] and CERN [15] could all be modified to track the initial collision excreta although probing these for neutrino flavor would require a secondary neutrino beam to collect appropriate interaction data. Clearly this is not a trivial task but may provide a means to allow neutrinos to remain in the standard model and still explain all observational measurement results.

\section{Discussion}


One can insist on a continuous, deterministic physical interpretation of what is happening to the neutrino during transport prior to measurement allowing a predictable oscillation behavior but the answer offered here is the same evolution occurring via an entangled set of particles. Examples of comparable deterministic looking physics with a quantum mechanism include magnetic resonance or the familiar 2 slit experiment. In the resonance behavior of electron paramagnetic resonance or nuclear magnetic resonance, the fermion spin is actually in only one of two states (parallel or anti parallel) but the superposition of both states allow a traditional precession based resonance realization following classical rules as a statistical average. With the two slit experiment, the wavefunction transports as a pure probability density function (a superposition of all possible states) obeying only wave mechanics during transmission requiring interference opportunities until measured.

The traditional derivation of neutrino oscillations assumes a right handed neutrino to exist giving rise to the Dirac mass term. That the derivation gives the right answer, does not necessarily require the interpretation of that derivation to be correct (similar to the Bohr model of the hydrogen atom). Rather, the argument offered here is that the probability evolution of the neutrino is constrained by its being entangled with its origins enabling flavor conservation. Here, all associated ejecta from the origin are all evolving through time which then forces the neutrino to likewise evolve to maintain the standard model conservation laws. Those particles which do not carry flavor then are unable to oscillate providing said test of the theory.

\section{Conclusions}

A new theory has been put forward which allows neutrinos to oscillate using only standard model physics where neutrino flavor is conserved. Effectively, the individual neutrino flavor changes are related back to being entangled with the combined system from which the neutrino originated (e.g., scattering or a decay process, etc). The flavor changes taking place in all associated particles emanating from the last interaction then becomes an experimentally testable feature of this model which would in principle, explain neutrino oscillations without a requirement for new physics. Tests could be comprised of measuring all flavors of excreta products to demonstrate the conservation of lepton flavor and parity expectation from the standard model.

\section{References}

1. Pallavicini, M. Solar neutrinos: experimental review and prospectives. J. Phys. Conf. Ser. 2015, vol. 598, no. 012007, doi:10.1088/1742-6596/598/1/012007

2. Dragoun, O. Review of direct neutrino mass experiments. AIP Conf. Proc. 2018, vol. 1686, no. 020008.

3. Fertl, M. Review of absolute neutrino mass measurements. Hyperfine Interac. 2018, vol. 259, no. 52, pp. 1-6.

4. Ehrlich, R. Review of the empirical evidence for superluminal particles and the $3+3$ model of the neutrino masses. Adv. Astron. 2019, Article ID 2820492, doi:10.1155/2019/2820492

5. Kisslinger, L. S. (2016) Review of neutrino oscillations with sterile and active neutrinos. Int. J. Mod. Phys.2016, vol. 31, no. 23, 1630037

6. Cai, Y. et al., From the trees to the forest: a review of radiative neutrino mass models. Front. Phys. 2017, vol. 5, doi10.3389/fphy.2017.00063

7. Studenikin, A. I. Electromagnetic neutrino: a short review. Nucl. Part. Phys. Proc. 2016, vol. 273-274, pp. 1711-1718

8. Suekane, F. Neutrino Oscillations A Practical Guide to Basics and Applications Springer, New York, 2015.

9. Griffiths, D. Introduction to Elementary Particles John Wiley \& Sons Inc. New York, 1987.

10. Verma, S. Theoretical and phenomenological status of neutrino physics: a brief review. Review Article. Adv. High Energy Phys., http://dx.doi.org/10.1155/2015/385968, 2015.

11. Bilenky, S. M. Review Paper. Neutrino masses, mixing and oscillations. Proc. Roy. Soc. London 2004, vol. 460, no. 2042, pp. 403443.

12. Singh, M. Testing texture two-zero neutrino mass matrices under current experimental scenario. EPL (Europhysics Letters). 2020, vol. 129, no. 1, 11002.

13. Abe, K., Abgrall, N., Aihara, H., Ajima, Y., Albert, J.B., Allan, D., Amaudruz, P.A., Andreopoulos, C., Andrieu, B., Anerella, M.D. and Angelsen, C., 2011. The T2K experiment. Nuclear Instruments and Methods in Physics Research Section A: Accelerators, Spectrometers, Detectors and Associated Equipment, 659(1), pp.106-135. 
14. Ahn, M.H., Aliu, E., Andringa, S., Aoki, S., Aoyama, Y., Argyriades, J., Asakura, K., Ashie, R., Berghaus, F., Berns, H.G. and Bhang, H., 2006. Measurement of neutrino oscillation by the K2K experiment. Physical Review D, 74(7), p.072003.

15. Adam, T., Agafonova, N., Aleksandrov, A., Altinok, O., Sanchez, P.A., Anokhina, A., Aoki, S., Ariga, A., Ariga, T., Autiero, D. and Badertscher, A., 2012. Measurement of the neutrino velocity with the OPERA detector in the CNGS beam. Journal of High Energy Physics, 2012(10), p.93.

16. O.W. Greenberg: Spin and Unitary Spin Independence in a Paraquark Model of Baryons and Mesons. Phys. Rev. Lett. 13, 598602 (1964) 